\title{
Multicommodity Flow and Circuit Switching
}

\author{
Tom Leighton* ${ }^{*} \quad$ Satish Rao ${ }^{\dagger} \quad$ Aravind Srinivasan ${ }^{\ddagger}$
}

\begin{abstract}
Given a set of request pairs in a network, the problem of routing virtual circuits with low congestion is to connect each pair by a path so that few paths use the same link in the network. We build on an earlier multicommodity flow based approach of Leighton 83 Rao to show that "short" flow-paths lead to path-selections with "low" congestion. This shows that such good path-selections exist for constant-degree expanders with "strong" expansion, generalizing a result of Broder, Frieze 83 Upfal. We also show, for infinitely many $n$, n-vertex undirected graphs $G_{n}$ along with a set $\mathcal{T}$ of connection requests, such that: (a) $\mathcal{T}$ is fractionally realizable using flow-paths that impose a (fractional) congestion of at most 1; but (b) any rounding of such a flow to the given set of flow-paths, leads to a congestion of $\Omega(\log n / \log \log n)$. This is progress on a long-standing open problem.
\end{abstract}

\section{Introduction}

Due to the rapid growth of high-speed integrated networks that provide vast bandwidth and support heterogeneous applications, considerable attention has been paid recently to edge-disjoint

\footnotetext{
* Mathematics Department and the Laboratory for Computer Science, Massachusetts Institute of Technology, Cambridge, MA 02139. E-mail: ftl@math.mit.edu.

${ }^{\dagger} \mathrm{NEC}$

Research Institute, 4 Independence Way, Princeton, NJ 08540. E-mail: satish@research.nj.nec.com.

$\ddagger$ Dept. of Information Systems \& Computer Science, National University of Singapore, Singapore 119260. Part of this work was done while visiting the NEC Research Institute, 4 Independence Way, Princeton, NJ 08540; part was supported by National University of Singapore Academic Research Fund Grants RP960620 and RP970607. E-mail: aravind@iscs.nus.edu.sg.
}

paths, bandwidth allocation, and related algorithmic problems on networks. We consider the problem of setting up low congestion "circuits" (paths) in a network. That is, given a set of requests pairs in a network, we wish to determine whether a set of paths can be routed between each request pair such that very few paths use the same wire. The natural linear relaxation of the circuit switching problem is a multicommodity flow problem. In this paper, we study the use of this relaxation to produce a solution to the circuit switching problem.

Suppose we are given a graph $G$ and a (set or multiset) $\mathcal{T}=\left\{\left(s_{i}, t_{i}\right): 1 \leq i \leq k\right\}$ of pairs of vertices of $G$. A sub-(multi-)set $\mathcal{T}^{\prime}$ of $\mathcal{T}$ is termed realizable if the pairs of vertices in $\mathcal{T}^{\prime}$ can be connected in $G$ by mutually edge-disjoint paths. The classical maximum disjoint paths problem is to find a realizable sub-(multi-)set of $\mathcal{T}$ of maximum cardinality. This is one of Karp's original NP-hard problems, and has resisted attempts at showing good approximability; the problem is NP-hard even when restricted to cubic planar graphs. Also, much of the difficulty in admission control and virtual-circuit routing in communication networks stems from the lack of good heuristics for the disjoint paths problem [9].

Raghavan and Thompson obtained nearly optimal results on the related problem where each edge can support a logarithmic number of paths rather than a constant number of paths: their idea is to solve the fractional version of the problem and then probabilistically "round" the solution to an integral solution [16]. We follow this outline in this paper.

An alternative method is based on using random walks in a graph to select candidate paths, and then to probabilistically select from this set. This method has been used by Broder, Frieze 
and Upfal [6] in the setting of expander graphs. They give a polynomial time algorithm that finds disjoint paths between any $O\left(n / \log ^{c} n\right)$ pairs of nodes in a sufficiently strong expander. Recently, they have used similar ideas to prove a better existential result [7]: the result is that any set of $c^{\prime} n / \log ^{2} n$ disjoint requests can be routed using edge-disjoint paths, where $c^{\prime}>0$ is a constant that depends only on the expansion properties of the graph. This technique has also been used by Broder, Frieze, Suen, and Upfal $[4,5]$ to give results that are constant factor of optimal for both node-disjoint and edge-disjoint paths on a certain set of random graphs (with nonconstant degree).

\subsection{Our Results}

Our work belongs to the more general area of rounding fractional flows to integral flows; by applying these results with the methods developed earlier in $[6,12]$, we get our results for expanders. So, we present our general rounding results first.

Suppose we are given a (directed or undirected) graph $G=(V, E)$ and a (set or multiset) $\mathcal{T}=\left\{\left(s_{i}, t_{i}\right): 1 \leq i \leq k\right\}$ of pairs of vertices of $G$. Let $|V|=n$ and $|E|=m$. We are required to connect each $\left(s_{i}, t_{i}\right)$ pair by one path in $G$, so that the congestion - the maximum number of paths using any given edge of $G$-is minimized. This is a well-known NP-hard problem with applications in routing and VLSI design. A well-known approach is to start with its multicommodity flow relaxation: finding the smallest positive real $\nu^{*}(\mathcal{T})$ such that we can ship one unit of flow from each $s_{i}$ to $t_{i}$ subject to no edge having to carry a total of more than $\nu^{*}(\mathcal{T})$ flow. That is, we relax the "integrality" constraint of either choosing one path or none at all for each $\left(s_{i}, t_{i}\right)$, to its fractional counterpart. This relaxation is a linear program and hence can be solved/approximated efficiently, and its optimum $\nu^{*}(\mathcal{T})$ is clearly a lower bound on the integral congestion $\nu(\mathcal{T})$.

How may we "round" a given fractional flow to a good integral flow, i.e., an integral flow of low congestion? In the positive direction, the following randomized rounding approach is well-known [16]. Given a fractional flow $\mathcal{F}$ of congestion (i.e., maximum total flow using any given edge) $C$, we may first decompose it into a set of at most $m$ flow paths, by standard "flow decomposition" [1]. In other words, we can efficiently construct, for each $i$, at most $m$ paths $P_{i, 1}, P_{i, 2}, \ldots$, each connecting $s_{i}$ to $t_{i}$ and with path $P_{i, j}$ carrying a flow $z_{i, j} \geq 0$. The "unit flow" condition implies that $\forall i, \sum_{j} z_{i, j}=1$; the congestion constraint implies that

$$
\forall f \in E, \quad \sum_{(i, j): f \in P_{i, j}} z_{i, j} \leq C .
$$

The standard randomized rounding approach is to pick, for each $i$, precisely one path $P_{i, j}$ : path $P_{i, j}$ is chosen with probability $z_{i, j}$, and the random choices for different $i$ are independent. By an application of the Chernoff bound, it can be seen that if, e.g., $C=1$, then the congestion resulting from randomized rounding is at most $O(\log n / \log \log n)$, with high probability; this can also be derandomized.

It is a major open question if such a congestion bound can be improved. Parameterizing the problem appropriately can help bring improvements. One such useful parametrization is that of the dilation $D$ of the fractional flow $\mathcal{F}$, defined to be the maximum length of any of the paths $P_{i, j}$. Improvements on the above Chernoff-based congestion bound when $D$ is $n^{o(1)}$, have been presented in [19]; these results work best when the fractional congestion $C$ is $\Omega(1)$. We complement this by working with the case where $C=o(1)$, which is the case, for instance, for routing with very low congestion on graphs. Our parameterization is also based on $D$, and our first main result is as follows, where $e$ denotes the base of the natural logarithm:

Theorem 1: Let $G$ be an arbitrary directed or undirected graph. Given a fractional flow $\mathcal{F}$ of congestion $C$ and dilation $D$, let $B$ be any positive integer such that $3(B+1) D C^{B} \leq B$ !. Then, there exists a way of rounding $\mathcal{F}$ wherein we choose one of the flow paths $P_{i, j}$ for each pair $\left(s_{i}, t_{i}\right)$, such that the maximum number of paths using any edge of the graph is at most $B$.

This theorem improves upon all similar theorems shown in [12]. It is also existentially optimal to within a constant factor for $B=1$ : it 
is not hard to construct instances of graphs and fractional flows wherein $C=\Theta(1 / D)$, such that there is no way of choosing one flow path for each $\left(s_{i}, t_{i}\right)$ with all the chosen paths being edgedisjoint. Note that $3(B+1) D C^{B} \leq B$ ! holds if $C \leq c_{0} B / D^{1 / B}$, where $c_{0}>0$ is an absolute constant. Thus, we get

Corollary 2: There is a constant $c_{0}>0$ such that the following holds. Let $G$ be any directed or undirected graph. Given a fractional flow $\mathcal{F}$ of congestion $C$ and dilation $D$, let $B$ be any positive integer such that $C \leq c_{0} B / D^{1 / B}$. Then, there exists a way of rounding $\mathcal{F}$ wherein we choose one of the flow paths $P_{i, j}$ for each pair $\left(s_{i}, t_{i}\right)$, such that the maximum number of paths using any edge of the graph is at most $B$.

Theorem 6 uses this corollary along with the work of Leighton \& Rao [12] to yield our improved routing results for expanders. Theorem 6 shows that for any constant-degree $n$-vertex expander $G$ with suitably strong expansion properties, there is a constant $c_{1}>0$ that depends only on the expansion of $G$ such that any set of at most $c_{1} n / \log ^{1+1 / B} n$ disjoint requests can be routed in $G$ with congestion at most $B$. This generalizes a corresponding result from [7] that works for the case of $B=1$ : it was shown in [7] that such expanders $G$ can support edge-disjoint paths for up to $c^{\prime} n / \log ^{2} n$ disjoint requests, where $c^{\prime}>0$ is a constant that depends only on the expansion of $G$.

Our second family of results are on the negative side. Recall from above that if $\nu^{*}(\mathcal{T}) \leq 1$, then $\nu(\mathcal{T})=O(\log n / \log \log n)$. It is a longstanding open question if this is tight; the only result known is that there exist families of instances for which $\nu^{*}(\mathcal{T}) \leq 1$ and $\nu(\mathcal{T}) \geq 2$. See $[18,13,14]$ for issues related to this question. It is very much an open question whether $\nu^{*}(\mathcal{T}) \leq 1$ implies that $\nu(\mathcal{T}) \leq 3$. We make progress on this question, by showing that for directed graphs, the gap of $O(\log n / \log \log n)$ is indeed tight. For undirected graphs, we show that this gap is tight for algorithms that round the fractional flow to the flow paths: i.e., algorithms that choose one of the flow paths $P_{i, j}$ for each $\left(s_{i}, t_{i}\right)$. This shows that for undirected graphs, the multicommodity flow has to be rerouted in some way, in order (if possible at all) to improve on the $O(\log n / \log \log n)$ gap, thus showing the problem of approximately minimizing congestion to be non-trivial in a technical sense:

Theorem 3: For each $n$ and any constant $c>0$, there exists a directed graph $G_{n}$ and an undirected graph $H_{n}$ with $n^{\Theta(1)}$ vertices each, such that the following holds. (a) There exist routing instances $\mathcal{T}$ on each of $G_{n}$ and $H_{n}$ such that there is a fractional flow of congestion at most $1 / \log ^{c} n$, and (b) any integral flow on $G_{n}$ for $\mathcal{T}$ has a congestion of $\Omega(\log n / \log \log n)$; any integral flow on $H_{n}$ where for each $\left(s_{i}, t_{i}\right)$, one of the paths $P_{i, j}$ is chosen, leads to a congestion of $\Omega(\log n / \log \log n)$.

The proofs of these rounding results are shown in Section 2; the application to low-congestion routing on expanders is discussed in Section 3.

\section{Proofs of Theorems}

We first recall the classical Lovász Local Lemma [8]:

Lemma 4 : (Lovász Local Lemma) Let $A_{1}, A_{2}, A_{3}, \ldots, A_{t}$ be events in an arbitrary probability space. Suppose that $A_{i}$ is mutually independent of all but $b$ other events $A_{j}$ for each $i \in\{1, \ldots, k\}$. Then if $e p(b+1) \leq 1$, we have $\operatorname{Pr}\left[\bigwedge_{i} \overline{A_{i}}\right]>0$.

Proof of Theorem 1: Fix $\mathcal{F}, B$ etc. Since $3(B+1) D C^{B} \leq B$ !, there is a sufficiently small but positive $\epsilon$ such that

$$
e(B+1) D C^{B} \leq(1-\epsilon)^{B+1} B ! .
$$

Fix such an $\epsilon>0$.

Recall that flow path $P_{i, j}$ is an $\left(s_{i}, t_{i}\right)$ path that carries a flow of value $z_{i, j} \geq 0$. For each $(i, j)$, we shall do the following. Let $z_{i, j}^{\prime}$ be the largest multiple of $\epsilon / m$ that is no larger than $z_{i, j}$; replace $P_{i, j}$ by $z_{i, j}^{\prime} m / \epsilon$ copies of itself, each of the copies carrying a flow of precisely $\epsilon / \mathrm{m}$. Since this process cannot increase any flow, it is clear that the fractional congestion on any edge is still at most $C$. How many flow paths do we have for each $\left(s_{i}, t_{i}\right)$ pair now? Recall, for each $i$, that 
$\sum_{j} z_{i, j}=1$, and that there are at most $m$ paths $P_{i, j}$. Now, since $z_{i, j}^{\prime} \geq z_{i, j}-\epsilon / m$, we see by summing that $\sum_{j} z_{i, j}^{\prime} \geq 1-m \epsilon / m=1-\epsilon$. Thus, since each of the new flow-paths carries a flow of exactly $\epsilon / m$, we have at least $m(1-\epsilon) / \epsilon$ flow paths for each $i$. In the rest of this proof, "flow paths" shall refer to these newly constructed flow paths $P_{i, j}^{\prime}$.

Our randomized rounding process is now simple: independently for each $\left(s_{i}, t_{i}\right)$ pair, pick one of its flow paths uniformly at random. We now use the Lovász Local Lemma to show that with positive probability, the congestion on any edge $f \in E$ is at most $B$. The crucial idea here is the right choice of "bad" events. For each edge $f \in E$ and every set of $B+1$ flow paths that use it, associate one bad event that says that all these $B+1$ paths get chosen by our random process. It clearly suffices to show that with positive probability, we avoid all of these bad events.

To use Lemma 4, we need to bound the probability of any given bad event, and bound the "dependence" $b$ in our system of bad events. Given a bad event, if two of the paths in it are for the same $\left(s_{i}, t_{i}\right)$ pair, the probability of the event is clearly 0 . Otherwise, since there are at least $m(1-\epsilon) / \epsilon$ flow paths for each $\left(s_{i}, t_{i}\right)$ pair, the probability of the event is at most

$$
p \doteq(\epsilon /(m(1-\epsilon)))^{B+1} .
$$

What about the "dependence" $b$ in the sense of Lemma 4? Recall that each bad event for us is a set of paths. Consider the bad event $\mathcal{E}$ corresponding to the set of paths $\left\{P_{i_{1}, j_{1}}^{\prime}, P_{i_{2}, j_{2}}^{\prime}, \ldots, P_{i_{B+1}, j_{B+1}}^{\prime}\right\}$. Since the random choices for different $\left(s_{i}, t_{i}\right)$ pairs are done independently, it is easy to see that $\mathcal{E}$ is independent of any combination of events of the form $\mathcal{E}^{\prime}=\left\{P_{r_{1}, s_{1}}^{\prime}, P_{r_{2}, s_{2}}^{\prime}, \ldots, P_{r_{B+1}, s_{B+1}}^{\prime}\right\}$, if $\left\{i_{1}, i_{2}, \ldots, i_{B+1}\right\} \cap\left\{r_{1}, r_{2}, \ldots, r_{B+1}\right\}=\phi$. So, suppose $r_{1} \in\left\{i_{1}, i_{2}, \ldots, i_{B+1}\right\}$, say $r_{1}=i_{1}$. For how many choices of $r_{2}, r_{3}, \ldots, r_{B+1}$ and $s_{1}, s_{2}, \ldots, s_{B+1}$ do we have a bad event of the form $\mathcal{E}^{\prime}$ ? Now, there are at most $m / \epsilon$ choices for $s_{1}$; fix any such choice. The path $P_{r_{1}, s_{1}}^{\prime}$ passes through at most $D$ edges, and each edge has at most $m C / \epsilon$ flow paths using it. Thus, the num- ber of bad events of the form $\mathcal{E}^{\prime}$ is at most

$$
(m / \epsilon) \cdot D \cdot\left(\begin{array}{c}
m C / \epsilon \\
B
\end{array}\right) \leq(m / \epsilon)^{B+1} D C^{B} / B ! .
$$

Similarly, for each $\ell$ such that $r_{\ell} \in\left\{i_{1}, i_{2}, \ldots, i_{B+1}\right\}$, the dependency is at most $(m / \epsilon)^{B+1} D C^{B} / B$ !. Thus, the total dependency is at most

$$
b \doteq(B+1)(m / \epsilon)^{B+1} D C^{B} / B !-1 .
$$

(The "-1" comes up since the event $\mathcal{E}$ would have been counted at least once in the above counting process.) Thus, by Lemma 4, (2), (3) and (4), we see that we avoid all the bad events with positive probability, i.e., there exists a rounding that makes the congestion at most $B$.

Proof of Theorem 3: We show the construction for $G_{n}$, and then sketch how to modify it for $H_{n}$. Let $k \leq n$ be a positive integer that divides $n$ (without loss of generality). Given a directed path $\left(v_{0}, v_{1}, \ldots, v_{n}\right)$ where there is an arc from each $v_{i}$ to $v_{i+1}$, we recursively construct $G_{n}$ as follows. Construct a new source vertex and a new sink vertex. Add arcs from the source vertex to the vertices $\left\{v_{i n / k}: 0 \leq i \leq k-1\right\}$, and add arcs from the vertices $\left\{v_{i n / k}: 1 \leq i \leq k\right\}$ to the sink vertex. The source and sink form a new demand pair. Now recurse separately on each of the $k$ directed paths $\left\{\left(v_{i n / k}, v_{i n / k+1}, \ldots, v_{(i+1) n / k}\right)\right.$ : $0 \leq i \leq k-1\}$. (The value of $k$ remains the same throughout, though the lengths of the chains change as we recurse.) We take $k$ to be $\log ^{c_{1}^{\prime}} n$, for some suitably large constant $c_{1}^{\prime}>$ 0 . Thus, the depth of the recursion is $d=$ $\Theta(\log n / \log k)=\Theta(\log n / \log \log n)$.

A fractional flow with low congestion is as follows. Let $s$ and $t$ denote the source and sink vertices respectively at the top level of the construction. Send $1 / k$ flow on each of the $k$ paths $\left(s, v_{i n / k}, v_{i n / k+1}, \ldots, v_{(i+1) n / k}, t\right)$, for $0 \leq i \leq$ $k-1$; the lower levels of the recursion can be handled the same way. Thus, since the congestion on any edge from each level of the recursion is at most $1 / k$, the maximum fractional congestion is at most $d / k$, which can be made smaller than $1 / \log ^{c} n$ by taking $c_{1}^{\prime}$ suitably large. 
What about integral flows? Let $s$ and $t$ be the source and sink vertices respectively at the top level of the construction. Note that any directed $(s, t)$-path must use all of the vertices in $\left(v_{i n / k}, v_{i n / k+1}, \ldots, v_{(i+1) n / k}\right)$, for some $i$. The idea now is to recurse on the path $\left(v_{i n / k}, v_{i n / k+1}, \ldots, v_{(i+1) n / k}\right)$. Continuing this way, we see that some edge must have congestion equaling the depth of the construction, i.e., congestion $d=\Theta(\log n / \log \log n)$.

Finally, the construction for $H_{n}$ is very similar, except that all the arcs above become undirected edges, and that all the directed fractional flow paths constructed above become undirected fractional flow paths.

\section{Results for Constant De- gree Expanders}

We start with some definitions.

\section{Definition 1:}

A graph $G=(V, E)$ is a $\beta$-expander graph if for any subset $S$ of $V$ where $|S|<|V| /|\beta|$ then size of $N(S)-S$ is at least $\min (\beta|S|,|V| /|\beta|)$.

\section{Definition 2:}

A graph $G=(V, E)$ is a triple expander graph if the edges $E$ can be partitioned into three sets $E_{1}, E_{2}$, and $E_{3}$ where $G_{1}=\left(V, E_{1}\right)$ and $G_{2}=$ $\left(V_{2}, E_{2}\right)$ and $G_{3}=\left(V, E_{3}\right)$ are expanders.

The above definitions are simplified for our convenience. Given a more general definition of expander one can show that any sufficiently good expander is a triple expander [6].

We now summarize some of the results of [12] as a theorem relevant for us:

Theorem 5: ([12]) Suppose we are given a routing problem with any set $\mathcal{T}$ of $k$ disjoint pairs of requests on a triple expander $G=(V, E)$; let $|V|=n$. Suppose, for some integer $B \geq 1$ and any routing request $\mathcal{T}^{\prime}$ on $G$, that a fractional flow for $\mathcal{T}^{\prime}$ with congestion $c_{2} k(\log n) / n$ and dilation $c_{3} \log n$ can be converted into an integral flow for
$\mathcal{T}^{\prime}$, with congestion at most $B\left(c_{2}, c_{3}>0\right.$ are constants that depend only on the expansion of $G$, and not on $n$ ). Then, $\mathcal{T}$ can be routed on $G$ with congestion at most $B$.

Substituting $C=c_{2} k(\log n) / n$ and $D=$ $c_{3} \log n$ in the statement of Corollary 2, we get our main result for expanders:

Theorem 6: Given any triple expander $G=$ $(V, E)$, let $|V|=n$. There is a constant $c_{1}>0$ that depends only on the expansion of $G$ and not on $n$, such that any set of at most $c_{1} n / \log ^{1+1 / B} n$ disjoint requests can be routed with congestion at most $B$.

As mentioned before, the case $B=1$ matches a result from [7], but our results for $B>1$ are new, and thus generalize the result of [7]. (It is shown in [7] that any set of up to $c^{\prime} n / \log ^{2} n$ disjoint requests can be routed in an edge-disjoint fashion-i.e., with congestion 1 -in $G$.) Moreover, we can make the above routing result constructive via the approach of $[3,2]$ by a losing a bit in the congestion bound; we shall present these results in the full version.

\section{Conclusions and Open Questions}

In this work, we have shown further progress on the routing capacity of suitably strong expander graphs under limited edge-capacity. A very interesting question is to study is the tightness of these results. It is not hard to check that any boundeddegree $n$-vertex graph can support edge-disjoint paths only for up to $O(n / \log n)$ pairs of connection requests. The results of [7] as well as ours, proven using very different means, give the lower bound of $\Omega\left(n / \log ^{2} n\right)$. Is this tight?

Another open question in the case of expanders is to come up with good constructive results for these problems, particularly for the case of edgedisjoint paths. As mentioned above, application of the Beck-Alon framework [3, 2] makes us lose a bit in the congestion bound; in particular, the case of congestion 1 (edge-disjoint paths) seems hard to handle by using this approach directly. In 
ongoing work, the authors are working on these issues and have made progress. It will also be of interest to develop good codes for these problems.

A tantalizing open question is the longstanding one of fractional vs. integral congestion. Our work shows that re-routing of fractional flows is necessary to improve on the worstcase relationship between fractional and integral congestion. Any progress on these questions should be of interest.

Acknowledgements. We thank the referees for their helpful comments.

\section{References}

[1] R. K. Ahuja, T. L. Magnanti, and J. B. Orlin. Network flows: theory, algorithms, and applications. Prentice Hall, Englewood Cliffs, New Jersey, 1993.

[2] N. Alon, A parallel algorithmic version of the Local Lemma, Random Structures and Algorithms, 2 (1991), pp. 367-378.

[3] J. Beck, An algorithmic approach to the Lovász Local Lemma, Random Structures and Algorithms, 2 (1991), pp. 343-365.

[4] A. Broder, A. Frieze, S. Suen and E. Upfal. Optimal Construction of Edge-Disjoint Paths in Random Graphs. In Proc. ACMSIAM Symposium on Discrete Algorithms, pages 603-612, 1994.

[5] A. Broder, A. Frieze, S. Suen and E. Upfal. Optimal Construction of Node-Disjoint Paths in Expander Graphs. In Proc. ACMSIAM Symposium on Discrete Algorithms, 1996.

[6] A. Broder, A. Frieze and E. Upfal. Existence and construction of edge-disjoint paths on expander graphs. SIAM J. Comput., 23:976989, 1994.

[7] A. Broder, A. Frieze and E. Upfal. Static and dynamic path selection on expander graphs: a random walk approach. In Proc.
ACM Symposium on the Theory of Computing, pages 531-539, 1997.

[8] P. Erdős and L. Lovász. Problems and results on 3-chromatic hypergraphs and some related questions. In Infinite and Finite Sets (A. Hajnal et. al. eds), pages 609-627, Colloq. Math. Soc. J. Bolyai 11, North Holland, Amsterdam, 1975.

[9] J. Kleinberg. Approximation algorithms for disjoint paths problems. PhD Thesis, Department of EECS, MIT, 1996.

[10] J. Kleinberg and É. Tardos. Disjoint paths in densely embedded networks. In Proc. IEEE Symposium on Foundations of Computer Science, pages 52-61, 1995.

[11] F. T. Leighton and S. B. Rao. An approximate max-flow min-cut theorem for uniform multicommodity flow problems with applications to approximation algorithms. In Proc. IEEE Symposium on Foundations of Computer Science, pages 422-431, 1988.

[12] F. T. Leighton and S. B. Rao. Circuit switching: a multi-commodity flow based approach. In Proc. Workshop on Randomized Parallel Computing, 1996.

[13] M. Lomonosov. Combinatorial approaches to multiflow problems. Discrete Applied Math., 11:1-94, 1985.

[14] M. Middendorf and F. Pfeiffer. On the complexity of the disjoint paths problem. Combinatorica, 13:97-107, 1993.

[15] D. Peleg and E. Upfal. Disjoint paths on expander graphs. Combinatorica, 9:289-313, 1989.

[16] P. Raghavan and C. D. Thompson. Randomized rounding: a technique for provably good algorithms and algorithmic proofs. Combinatorica, 7:365-374, 1987.

[17] A. Schrijver. Homotopic routing methods. In B. Korte, L. Lovász, H. J. Prömel, and A. Schrijver, Editors, Paths, Flows, and VLSI-Layout, pages 329-371, SpringerVerlag, Berlin, 1990. 
[18] P. D. Seymour. On odd cuts and planar multicommodity flows. J. London Math. Soc., 42:178-192, 1981.

[19] A. Srinivasan. An extension of the Lovász Local Lemma, and its applications to integer programming. In Proc. ACM-SIAM Symposium on Discrete Algorithms, pages 6-15, 1996. 